\title{
Thuringopteryx - eine „permische“ Eintagsfliege im Buntsandstein (Insecta, Pterygota)
}

\author{
Thuringopteryx, a "Permian“ mayfly in the Buntsandstein (Insecta, Pterygota)
}

RAINER WILLMANN, Göttingen

mit 3 Abbildungen

\begin{abstract}
WILlMANN, R. 2008. Thuringopteryx - eine „permische“ Eintagsfliege im Buntstandstein (Insecta, Pterygota). Paläontologische Zeitschrift 82 (1): 95-99, 3 Abb., Stuttgart, 31. 3. 2008.

Kurzfassung: Bisher waren die Misthodotida, ein monophyletisches Stammgruppentaxon der Ephemeroptera, nur aus dem Perm nachgewiesen. Thuringopteryx gimmi KuHN, 1937, meist als eine Art der Odonatoptera, Palaeodictyoptera oder Saltatoria angesehen, ist jedoch ebenfalls ein Stammgruppenvertreter der Eintagsfliegen ähnlich Misthodotes. Sie stimmt mit den anderen Misthodotida in ihrem einästigen Cubitus anterior synapomorph überein, mit $M i s$ thodotes außerdem in ihrer kurzen Subcosta. Somit haben die Misthodotida zumindest bis in das frühe Mesozoikum überlebt. Die Verwandtschaftsbeziehungen von Misthodotes und seinen näheren Verwandten werden erörtert.
\end{abstract}

Schlüsselwörter: Ephemeroptera • Misthodotida • Phylogenie • Trias • Deutschland

Abstract: The Misthodotida have been known from the Permian only. However, Thuringopteryx gimmi KuHN, 1937 from the middle Buntsandstein of Thuringia, Germany, usually considered to be a representative of the Odonatoptera, Palaeodictyptera or Saltatoria-Ensifera, belongs in the stem group of the mayflies, sharing with the Misthodotida a one-branched CuA and with Misthodotes a short Sc. Hence, the Misthodotida survived at least until the early Mesozoic. The phylogenetic relationships of Misthodotes and its closest allies are outlined.

Keywords: Ephemeroptera $\bullet$ Misthodotida $\bullet$ phylogeny $\bullet$ Triassic $\bullet$ Germany

\section{Einleitung}

Im Perm gab es eine Reihe von Stammgruppenvertretern der Eintagsfliegen, die sich im Gegensatz zu den rezenten Arten durch vier etwa gleich große, im Umriss schlank-elliptische Flügel auszeichneten, bei denen die Mundwerkzeuge sowie der Prothorax noch gut ausgebildet waren und deren Larven auf den ersten neun Abdominalsegmenten je ein Paar Tracheenkiemen trugen und mehrgliedrige Tarsen hatten. Das Flügelgeäder wies eine wenig markante und nur geringfügig gebogene Costalstrebe oder -brücke auf, und die beiden Cubitaladern verliefen einander annähernd parallel, d. h. sie divergierten nicht in Richtung Flügelrand. Die heutigen Eintagsfliegen sehen sehr anders aus: Die Vorderflügel sind zu den Hauptflugorganen geworden und von annähernd dreiseitigem Umriss, die Hinterflügel sind reduziert; dementsprechend ist auch der Mesothorax sehr kräftig entwickelt, während der Metathorax nur klein ist. Auch der Prothorax ist nur klein. Die Mundwerkzeuge sind reduziert, denn die Subimagines und Imagines nehmen keine Nahrung mehr zu sich. Am Vorderrand der Flügel findet sich nahe der Flügelbasis die Costalstrebe als nun recht auffällige bogenförmige Struktur. Die Larven haben allenfalls auf den ersten sieben Abdominalsegmenten Tracheenkiemen, und ihre Tarsen sind eingliedrig.

Die so charakterisierten Stammgruppenvertreter der Eintagsfliegen wurden von TILLYARD (1932) als „Permoplectoptera“ zusammengefasst, eine wahrscheinlich paraphyletische Gruppierung. Sie umfasste zunächst die Protereismatidae und die Misthodotidae (letztere = Eudoteridae, siehe CARPENTER 1979: 282). Später fügten andere Autoren weitere Taxa hinzu und kreierten Namen wie Protereismatoidea und Protereismatina, die aber sämtlich, wenn sie lediglich dem Um-

Anschrift des Verfassers: Rainer Willmann, Institut für Zoologie und Anthropologie und Zoologisches Museum der Universität Göttingen, Berliner Str. 28, D-37073 Göttingen, Deutschland; e-mail <rwillma1@gwdg.de>. 
fang der Permoplectoptera sensu TILLyARD entsprachen, redundant waren. Protereismatidae und Misthodotidae sind von verschiedenen Fundorten der heutigen Nordhemisphäre beschrieben worden, alle aus dem unteren Perm: Nordamerika, westliches Russland, Mitteleuropa. Von beiden Taxa nahm man an, dass sie noch im Paläozoikum ausgestorben wären.

Die Protereismatidae (nur Protereisma SELLARDS, 1907) zeichneten sich durch Flügel mit langer Subcosta und langem Radiussektor aus (das heißt, sowohl Sc als auch RS reichten bis fast an den Flügelapex), und ihr Cubitus anterior wies eine Triade auf. Die Misthodotidae (bisher nur Misthodotes SELLARDS, 1909, auch wenn manche Arten unter anderen Gattungsnamen geführt worden waren; CARPENTER 1979, 1992) hatten eine kürzere Subcosta, einen kürzeren Radiussektor, und CuA war - von vereinzelt vorkommenden terminalen Seitenzweigen abgesehen - einfach. Die Ausprägung bei Protereisma findet sich auch bei den rezenten Eintagsfliegen; dies ist als Symplesiomorphie anzusehen.

Der einfache $\mathrm{CuA}$ ist eine Konvergenz zu den rezenten Eintagsfliegen. Eine Reihe diesen näher stehenden Stammgruppenvertreter hatten noch eine $\mathrm{CuA}$ Triade, z.B. Palingeniopsis, Hexagenites, Ephemeropsis oder Cratogenitoides.

Es gibt aber noch eine Art, die diesen Stammgruppenvertretern sehr ähnlich ist. Sie stammt aber weder aus dem Perm noch wurde sie bisher allgemein als Eintagsfliege anerkannt. Es handelt sich um Thuringopteryx gimmi KuHN, 1937 aus dem Buntsandstein Thüringens. Thuringopteryx war zunächst nicht sehr genau beschrieben worden und wurde daher von MÜLLER (1965) neu untersucht. Er hielt sie wie KUHN für den Flügel eines Vertreters der „Protodonata“. ZEUNER (1939) und CARPENTER (1992: 176) sahen in Thuringopteryx eine Saltatorie (Ensifera incertae familiae), was damit zusammenhängen könnte, dass KuHN die Existenz eines recht ausgedehnten Präcostalfeldes angenommen hatte. BECHLY (1997) hingegen glaubte, es sei eine Palaeodictyoptere (eventuell Spilapteridae) und damit eine triassische Art dieses ansonsten nur aus dem Paläozoikum bekannten Taxons (siehe unten), während GRIMALDI \& ENGEL (2005: 165) sich am ehesten eine Zugehörigkeit zu den Eintagsfliegen vorstellen konnten. Darin folgten sie KLUGE \& SINITSHENKOVA (in RASNITSYN \& QUICKE 2002: 95) bzw. KLUGE (2004: 359), der Thuringopteryx als Euephemeroptera inc. sedis aufgelistet hatte.

Die widersprüchlichen Interpretationen hängen mit den bisher verfügbaren unvollständigen Informationen über Thuringopteryx zusammen. Aus diesem Grunde sei eine erneute Beschreibung gegeben.

\section{Systematische Paläontologie}

\author{
Thuringopteryx gimmi KUHN, 1937 \\ Abb. 1, 2
}

Holotypus (Nr. 31/2 a und b [nach einem später beigefügten, per Schreibmaschine verfassten Zettel 37/2 a, b], Geologische Sammlung der Technischen Universität/Bergakademie Freiberg): Ein fast vollständiger Flügel, 1,7 cm lang (Positiv), mit fragmentärem Gegenstïck (Negativ).

Fundort: Singen bei Stadtilm/Thüringen, mittlerer Buntsandstein [sm3, Lettenlinse im Chirotheriensandstein]).

Beschreibung: Costa nahe der Flügelbasis am Vorderrand mit einer Reihe von Ansatzstellen von Borsten, die als dunkle Punkte erhalten sind (ähnlich wie das CARPENTER 1979 für Protereisma darstellte); von Borsten findet sich ansonsten keine Spur. $\mathrm{C}$ und Sc verschmelzen nach etwa $2 / 3$ der Flügellänge. Sie bilden gemeinsam bis kurz vor dem Flügelapex in Fortsetzung der Costa ein kräftiges distad sich verjüngendes Aderstuick. $\mathrm{R}$ und Rs, soweit in Richtung Flügelbasis verfolgbar, getrennt. Rs mit sieben dominierenden Längsadern: $\mathrm{RsA}_{1} ; \mathrm{RsA}_{2}$ (mit 4 Ästen); iRs, RsP. RsA $A_{2}$ trennt sich kurz vor der Flügelmitte von $\mathrm{RsA}_{1}$. Etwas weiter proximal liegt die RsA/RsP-Gabel. Von RsP zweigt unmittelbar danach (das heißt in Richtung Flïgelapex) iRs ab. MA mit 3 Längsästen; läuft in Richtung Flügelbasis auf Rs zu (die anzunehmende Stelle der Fusion beider ist beschädigt). Der Stamm von MA geht ohne Knick in $\mathrm{MA}_{1}$ über; diese beiden Aderabschnitte zusammengenommen verlaufen daher annähernd geradlinig. CuA einfach.

Die hinter $\mathrm{CuA}$ folgenden und nur schlecht erhaltenen Adern sind schwer zu interpretieren. An der Flügelbasis ist CuP gut erkennbar, doch ist sein Verlauf in Richtung Flügelrand nicht ununterbrochen überliefert. Da CuP bei allen Ephemeroptera einfach ist, könnte die kurze Ader hinter CuP (nahe dem Flügelhinterrand) zur 1. Analis gehören. Eventuell aber gehört sie noch $z u$ $\mathrm{CuP}$ (ähnlich wie ein vor CuP liegender Zweig bei Protereisma directum von CARPENTER 1979 noch zu CuP gerechnet wurde). Zur 1. Analis gehört offenbar das darauf folgende längere Aderstuick.

Anmerkungen: Wahrscheinlich auf Grund von Beschädigungen ist von der Costalbrücke nichts zu erkennen. Im distalen Bereich des Costalfeldes (zwischen Sc und C) sind zwei Queradern sichtbar. Im Costalfeld von Protereisma und Misthodotes finden sich hingegen zahlreiche Queradern. Es ist daher möglich, dass bei Thuringopteryx weitere Queradern in diesem Teil lediglich aus Erhaltungsgründen nicht vorhanden sind. Ob es sich um einen Vorder- oder Hinterflügel handelt, ist wegen der großen Ähnlichkeit zwischen den meso- und metathorakalen Flügeln vieler Stammgruppenvertreter der Eintagsfliegen (darunter auch denen der Misthodotida) ohne weiteres Fundmaterial nicht zu bestimmen.

Auf folgende Ungenauigkeiten in der Darstellung von MÜLLER (1965, dort vor allem Abb. 2) sei besonders hingewiesen, um für die Ermittlung der taxischen 

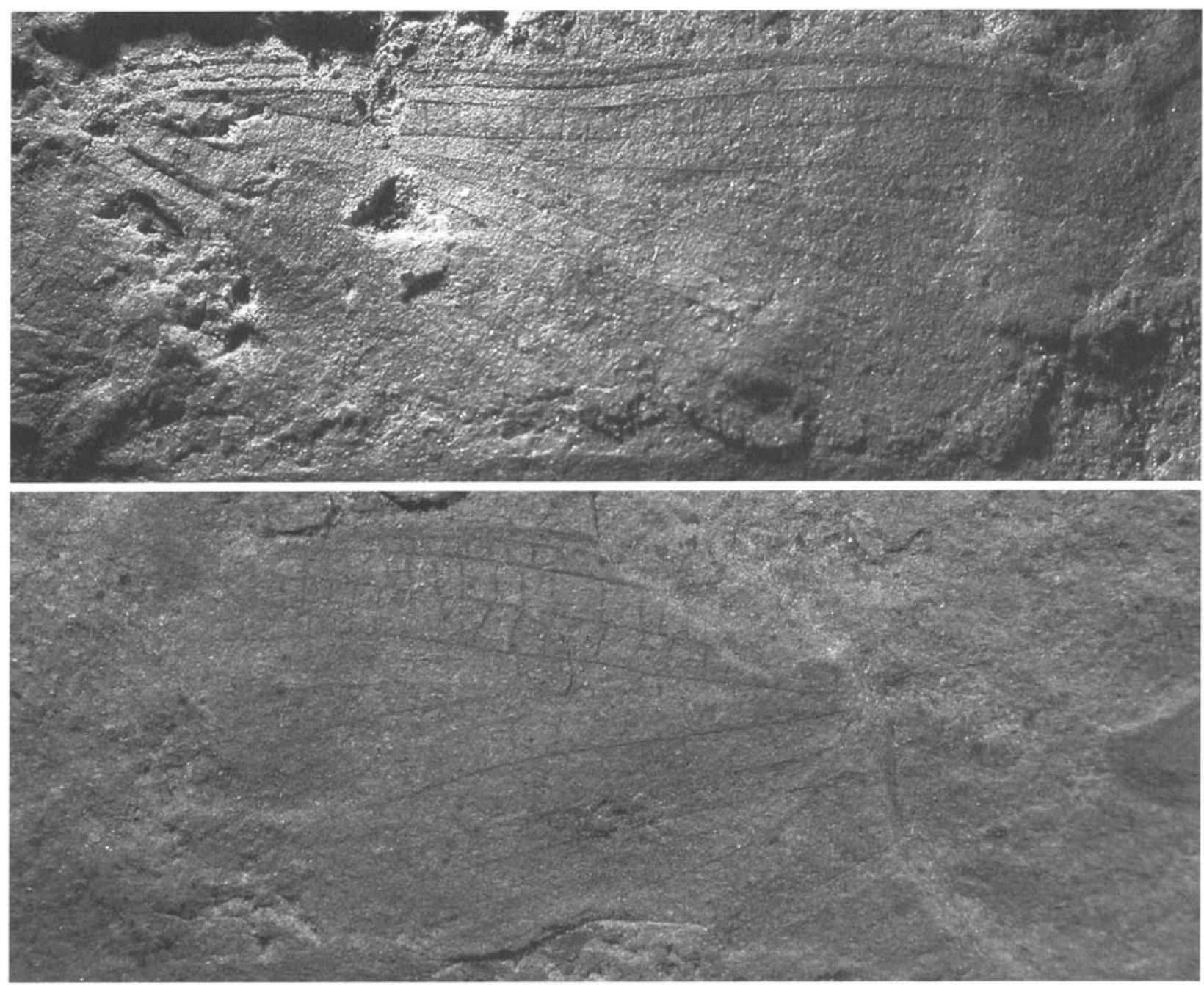

Abb. 1. Thuringopteryx gimmi KuHN, 1937, Holotypus, Positiv und Negativ.

Zugehörigkeit des Flügels wichtige Widersprüche zur hiermit vorgelegten Beschreibung darzulegen: Der distale Flügelrand ist recht gut erhalten. Die Subcosta verschmilzt viel früher als von MüLLER angegeben mit der Costa (hier ist die Darstellung von KUHN 1937 richtig). Der Radius biegt nicht in den apikalen Flügelbereich hinein, sondern trifft nach geradem Verlauf auf den Flügelrand. Die Abstände zwischen Sc und R und R und $\mathrm{RS}_{1}$ sind im distalen Flügelteil größer als von MÜLLER dargestellt. Struktur und Verlauf von RS und MA sind von MÜLLER im Wesentlichen korrekt wiedergegeben worden, doch sind nahe dem Flügelrand sowohl zwischen $\mathrm{RS}_{6-7}$, zwischen $\mathrm{RS}_{7}$ und $\mathrm{MA}_{1}$ als auch zwischen den drei MA-Adern kurze Intercalaradern erkennbar. An der Flügelbasis hat MÜLLER im Zusammenhang mit dem Cubitus ein Aderstück mehr abgebildet als tatsächlich vorhanden ist (MüLLER wies selbst darauf hin, dass er nicht alle Queradern eingetragen habe).

\section{Vergleiche}

Eine relativ kurze Sc findet sich auch bei Misthodotes. Dies steht im Gegensatz zu allen anderen paläozoischen und zum Grundmuster der rezenten Eintagsfliegen. Rs hat bei Thuringopteryx im Gegensatz zu Misthodotes Intercalaradern auch $\mathbf{z w i s c h e n ~ d e n ~ v o r d e r e n ~ d r e i ~ L a ̈ n g s - ~}$ adern. Zwischen den drei dominierenden MA-Ästen ebenfalls mit Intercalaradern (ebenfalls im Unterschied zu Misthodotes). Bei mehreren Misthodotes-Arten besteht wie bei Thuringopteryx die Tendenz, dass der MAStamm ohne Biegung in $\mathrm{MA}_{1}$ übergeht (derartige Biegungen bestehen häufig an den Aufspaltungen der $\mathrm{Me}$ dia anterior in $\mathrm{MA}_{1} / \mathrm{MA} 2$ sowie der Gabelung $\mathrm{MA}_{1}$ ' iMA). Der CuA ist wie bei Misthodotes einästig (Unterschied zu Protereisma und anderen). Diese Eigenschaften erlauben eine genauere Bestimmung der Verwandtschaftsbeziehungen innerhalb der Stammgruppe der Eintagsfliegen, wobei zunächst auf die ursprüngliche Beschreibung der Misthodotidae eingegangen werden muss. Ältere wahrscheinliche Stammgruppenvertreter der Eintagsfliegen wie Lithoneura oder Syntonoptera können wegen ihres wesentlich ursprünglicheren Charakters in diesem Zusammenhang außer Betracht gelassen werden (siehe hierzu WILLMANN 2007).

TILlyARD (1932) kennzeichnete die Misthodotidae wie folgt (in diesem Zusammenhang sind nur die Flügel- 


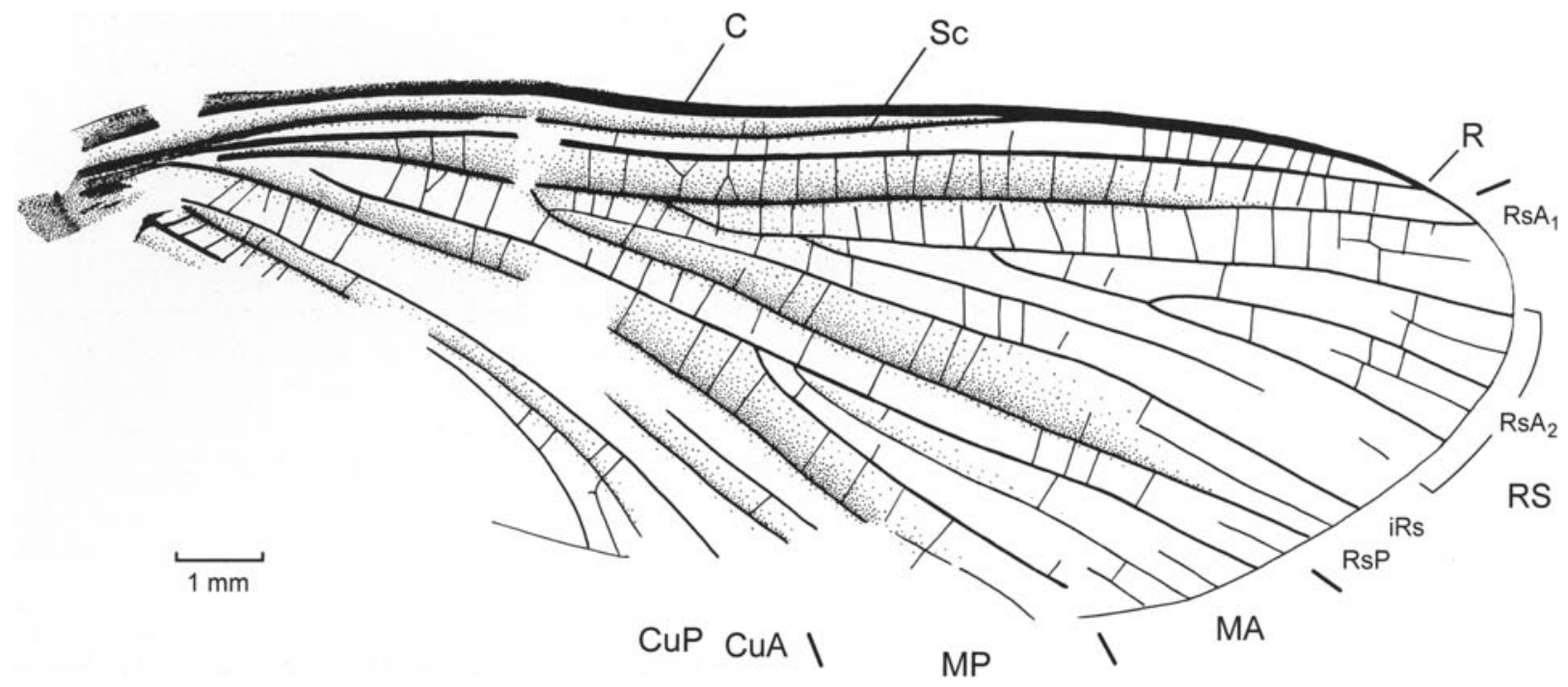

Abb. 2. Thuringopteryx gimmi KUHN, 1937, Holotypus, Positiv.

strukturen von Relevanz): Vorderflügel relativ breiter als bei Protereisma, Hinterflügel breiter als der Vorderflügel. Costal- und Subcostalfeld breiter als bei Protereis$m a$, mit weniger und in weiterem Abstand voneinander liegenden Queradern. Sc und R enden erheblich weiter vor dem Flügelapex als bei Protereisma. $\mathrm{CuA}$ nicht gegabelt (ohne Triade), allenfalls mit terminalen Zweigen (TILlyARD 1932: 260). Zu den Misthodotidae wurde bisher nur der unterpermische Misthodotes (= Eudoter, siehe CARPENTER 1979: 282) gerechnet (Typus generis: $M$. obtusus (SELLARDS, 1907)).

Durch seine Beschreibung der Misthodotidae schränkte TILLYARD implizit ein, welche Arten in dieses Taxon einzubeziehen seien und welche nicht. Arten mit langer Subcosta (ähnlich der von Protereisma) können somit, wenn es sich, wie anzunehmen ist, um eine Plesiomorphie handelt, nicht zu den Misthodotidae gehören.

Andererseits aber gab es Arten, die ursprüngliche Eigenschaften, wie sie bei Protereisma beibehalten wurden, und abgeleitete Merkmale, wie Misthodotes sie zeigt, kombinierten oder bei denen die Ausprägung mancher Strukturen zwischen denen von Protereisma und denen von Misthodotes vermittelten. TILLYARD waren sie, da später entdeckt, nicht bekannt. Sie hatten eine relativ lange Subcosta und einen einfachen $\mathrm{CuA}$. Es handelt sich um die Arten stapfi KINZELBACH \& LUTZ, 1984 aus dem Unter-Perm von Südwestdeutschland und zalesskyi TSHERNOVA, 1965 aus dem Unter-Perm von Russland, bei denen sich Sc noch ziemlich weit in den apikalen Flügelbereich hineinkrümmt.

Nach TILLYARDS Charakterisierung der Misthodotidae und unter Berücksichtigung der vorstehenden Ausführungen über die ursprüngliche bzw. abgeleitete Ausprägung der einzelnen Flügelstrukturen sind diese beiden Arten nicht zu diesem Taxon und erst recht nicht zu Misthodotes zu rechnen. WILLMANN (2007) führte für sie daher die Namen Arnulfias (Typusart: stapfi KIN-
ZELBACH \& LUTZ) und Eurekter (Typusart: zalesskyi TSHERNOVA) ein.

Aufgrund der verfügbaren Merkmale zeichnen sich folgende Verwandtschaftsbeziehungen ab (Abb. 3): $A r$ nulfias stapfi und Eurekter zalesskyi stimmen mit den

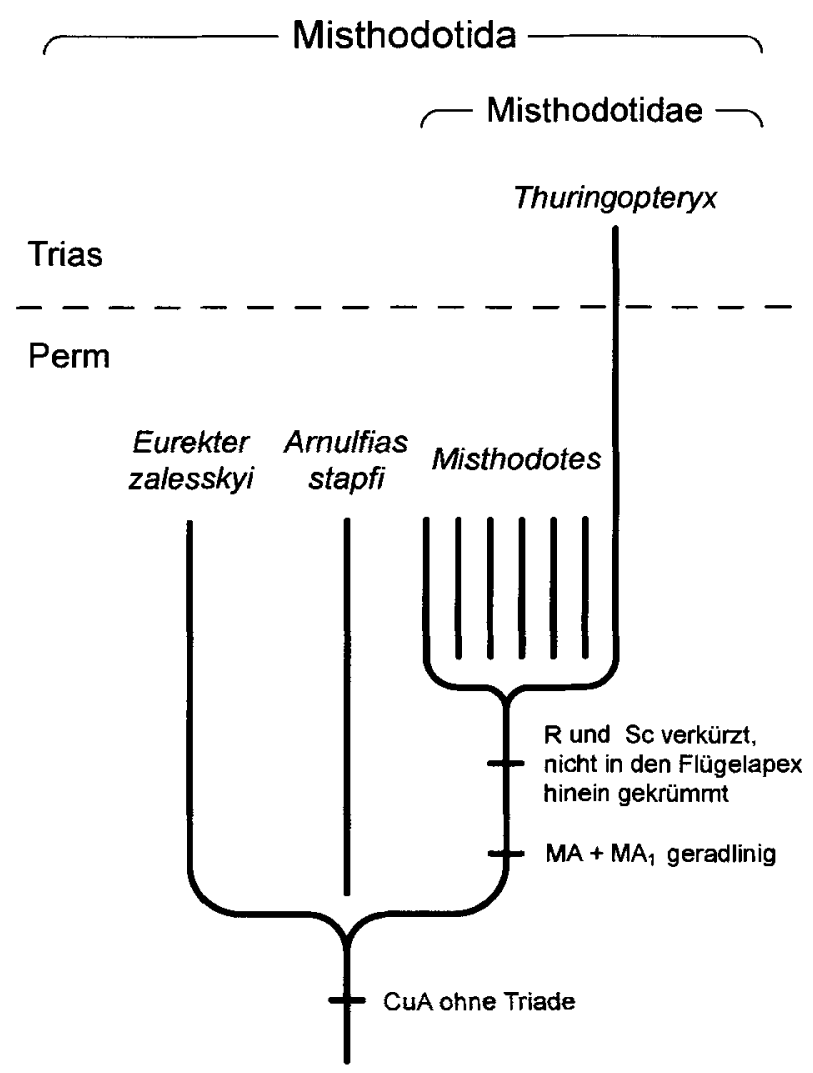

Abb. 3. Verwandtschaftsbeziehungen innerhalb der Misthodotida. Thuringopteryx als nächster Verwandter von Misthodotes; Misthodotes ist möglicherweise paraphyletisch (WILLMANN 2007). 
Misthodotidae im einästigen CuA synapomorph überein (zusammen: Misthodotida WILLMANN, 2007). Die Schwestergruppenbeziehungen zwischen Arnulfias, Eurekter und den Misthodotidae sind unklar.

Bei den Misthodotidae sind Sc und R verkürzt, und beide Adern laufen nicht unter einer dem Flügelrand folgenden Krümmung in den Flügelapex hinein. Diese Merkmale werden als Autapomorphien interpretiert. Bei den meisten Flügeln von Misthodotes und auch Thuringopteryx besteht die Tendenz, dass der Stamm von MA ohne jeden Knick in MA $_{1}$ übergeht, $\mathbf{M A}-\mathbf{M A}_{1}$ bilden somit eine annähernd geradlinige Struktur inmitten des Flügels, von der die beiden hinteren MA-Äste abzweigen. Ob dies von systematischer oder phylogenetischer Signifikanz ist, ist ungewiss; es kann sich dabei wie bei rezenten Arten um individuelle Varianten handeln.

Für die Misthodotes-Arten (edmundsi CARPENTER, 1979, biguttatus TILlYARD, 1932, delicatulus TILLYARD, 1932, ovalis TILLYARD, 1932, sharovi TSHERNOVA, 1965 und obtusus SELLARDS, 1909; Flügel aller dieser Arten sind bei KINZELBACH \& LUTZ, 1984: Abb. 1 im Vergleich zusammengestellt) lassen sich keine Synapomorphien feststellen. Misthodotes in diesem Umfang ist somit möglicherweise nicht monophyletisch. Auffällig ist aber das weitgehende Fehlen von Intercalaradern bei diesen Arten.

Als Autapomorphie von Thuringopteryx ist die gegenüber Misthodotes weitergehende Verkürzung von Sc $\mathrm{zu}$ nennen. Ungewöhnlich ist ihre Struktur von $\mathrm{RsA}_{2}$. Vielleicht ist sie nicht für Thuringopteryx gimmi als Art charakteristisch, sondern lediglich eine individuelle Variante. Zur phylogenetischen Position der Misthodotida siehe WILLMANN (2007).

\section{Kommentare zu früheren Verwandtschaftsannahmen}

ZEUNER (1939) hielt Thuringopteryx für eine Ensifere (inc. sedis, siehe auch CARPENTER 1992). Diese Annahme beruhte auf der Originalbeschreibung von KUHN (1937, siehe auch SCHMIDT 1938: 47) und kann hier unerörtert bleiben. Auf Grund der teilweise besseren Darstellung von MÜLLER (1965) schloss BECHLY (1997: 53), dass es sich weder um eine Odonatoptere noch um eine Ephemeroptere handeln könne, da die Intercalaradern IR 1 und IR2 fehlen. Vielmehr sei Thuringopteryx eine Palaeodictyoptere, wahrscheinlich aus der engeren Verwandtschaft um die Spilapteridae. "This is", so BECHLY (1997: 53) "quite remarkable, since previously there were no Triassic palaeodictyopteroids known at all“. BECHLY konnte nicht wissen, dass auch die Darstellung von MÜLLER zu Fehlschlüssen verleitet und dass Intercalaradern in erheblicher Anzahl vorhanden sind. Wenn es sich nun um eine Eintagsfliege mit charakteristischen Eigenschaften der Misthodotidae und
Protereismatidae handelt, so ist dies aber nicht weniger bemerkenswert, denn auch für diese relativ ursprünglichen Formen hatte man bisher ein Erlöschen noch im Perm angenommen.

\section{Dank}

Jörg Schneider, Freiberg, machte mir in unkomplizierter Weise und innerhalb kürzester Zeit den Holotypus von Thuringopteryx gimmi zugänglich, dafür ein herzliches Dankeschön! Für Anmerkungen zum Manuskript bin ich Jes Rust, Bonn, und einem anonymen Gutachter sehr verbunden.

\section{Literatur}

BECHLY, G. 1997. New fossil odonates from the upper Triassic of Italy, with a redescription of Italophlebia gervasuttii Whalley, and a reclassification of Triassic dragonflies. - Rivista dell Museo Civico di Science Naturali "Enrico Caffi" Bergamo 19: 31-70.

CARPENTER, F.M. 1933. The Lower Permian Insects of Kansas. Part 6. - Proceedings of the American Academy of Arts 68: 411-503.

CARPEnTER, F.M. 1979. Lower Permian insects from Oklahoma. Part 2. Orders Ephemeroptera and Palaeodictyoptera. - Psyche 86: 261-290.

CARPENTER, F.M. 1992. Superclass Hexapoda. Treatise on Invertebrate Paleontology R, Arthropoda 4 (3-4). - 655 S., Boulder \& Lawrence (Geological Society of America and University of Kansas Press).

GRIMALDI, D. \& ENGEL, M. 2005. Evolution of Insects. - 755 S.. Cambridge (Cambridge University Press).

KinZELBACH, R. \& LUTZ, H. 1984. Eine neue Eintagsfliege Misthodotes stapfi n. sp. aus dem Rotliegenden des Nahe-Gebietes (Ephemeroptera: Permoplectoptera: Misthodotidae). - Paläontologische Zeitschrift 58: 247-253.

KLUGE, N. 2004. The Phylogenetic System of Ephemeroptera. - 442 S., Dordrecht (Kluwer).

KuHN, O. 1937. Insekten aus dem Buntsandstein von Thüringen. - Beiträge zur Geologie Thüringens 4: 190-193.

Müller, A.H. 1965. Insektenreste aus der Trias (Buntsandstein, Keuper) von Thüringen. - Geologie 14: 865-877.

RASNITSYN, A. \& QUICKE, D.L. 2002. History of Insects. - 517 S., Dordrecht (Kluwer).

SCHMIDT, M. 1938. Die Lebewelt unserer Trias. Nachtrag. - 143 S., Oehringen (F. Rau).

TillyaRD, R.J. 1932. Kansas Permian Insects. Part 15. The Order Plectoptera. - American Journal of Science 223: 97-134, 237272.

TSHERNOVA, O. 1962. Otrjad Ephemeroptera Podenki. - In: RODENDORF, B., Hrsg., Osnovy paleontologii: 55-64, Moskva (Akademija Nauk SSSR).

TSHERnova, O. 1965. Some fossil Mayflies (Ephemeroptera, Misthodotidae) from Permian beds of the Ural. - Entomological Review 44: $202-207$

TSHERNOVA, O.A. 1970. On the classification of Fossil and Recent Ephemeroptera. - Entomological Review 49: 71-81.

WILlmaNN, R. 2007. Die Stammgruppenvertreter der Ephemeroptera und ihre systematische Stellung. - Species, Phylogeny and Evolution 1: 108-132.

ZEUNER, F. 1939. Fossil Orthoptera Ensifera. - 321 S., London (British Museum of Natural History).

Manuskripteingang / manuscript received 27. 4. 2007; Manuskriptannahme / manuscript accepted 23. 1. 2008. 